\title{
EFFECT OF MANNITOL STRESS ON MORPHOLOGICAL, BIOCHEMICAL AND POLYPHENOL PARAMETERS IN BROCCOLI SPROUTS (BRASSICA OLERACEA VAR. ITALICA)
}

\author{
KIANI, S. - BABAEIANJELODAR, N.* - BAGHERI, N. - NAJAFIZARRINI, H. \\ Department of Plant Breeding, Sari Agricultural Sciences and Natural Resources University \\ Sari, Mazandaran, Iran \\ *Corresponding author \\ e-mail:n.babaeiyan@umz.ac.ir \\ (Received $29^{\text {th }}$ Oct 2017; accepted $13^{\text {th }}$ Mar 2018)
}

\begin{abstract}
To assess the effect of drought stress induced by mannitol at three levels $(0,88$ and $176 \mathrm{mM})$ on biochemical and polyphenolic traits of six $F_{1}$ broccoli hybrids, a factorial experiment based on completely randomized design in three replications was implemented at research station of Agricultural Sciences and Natural Resources University, Sari, Mazandaran, Iran in 2016. The results showed that mannitol stress reduced dry weight and shoot length significantly for all varieties but with a different rate. Total phenolic, flavonoid, and anthocyanin contents, the activity of enzymatic antioxidants, and DPPH activity were significantly higher under $176 \mathrm{Mm}$ mannitol application condition than control. In addition, results showed that mannitol stress increased the content of sulforaphane. Marathon genotype showed the highest content of sulforaphane among all varieties under both normal (6.139) and under stress (14.122) conditions. Marathon and Heraklion genotypes could be suggestively used for breeding program to increase content of sulforaphane coupled with other traits. Since the content of sulforaphane along with phenolic compounds and antioxidant activities were higher under both severe and moderate stress conditions, using moderate mannitol stress treatment can be implemented for increasing the content of these suitable compounds in broccoli.
\end{abstract}

Keywords: sulforaphane, DPPH, principal component, flavonoid, malondialdehyde

\author{
Abbreviations \\ ROS Reactive oxygen species \\ $\mathrm{H}_{2} \mathrm{O}_{2} \quad$ Hydrogen peroxide \\ $\mathrm{O}_{2} \quad$ Superoxide radicals \\ MDA Malondialdehyde \\ SOD Superoxide dismutase \\ APX Ascorbic peroxidase \\ POX Guaiacul peroxidase \\ CAT Catalase \\ DPPH 2, 2-diphenyl-1-picrylhydrazyl
}

\section{Introduction}

Broccoli (Brassica oleracea var. Italica) is a member of the Brassicaceae family and its wild form is found throughout the Mediterranean region and is widely cultivated in many countries of Europe and America along with Asian countries (Abou El-Magd et al., 2013). Broccoli is one of the highly valuable vegetable that is rich in nutrients content and it has many benefits for human health. Broccoli sprouts contain a substance called sulforaphane which intensely reduces the number, size, and proliferation of cancerous tumors; and moreover the sprout is rich in fiber, carotenoids, vitamin A, vitamin C and vitamin K (Beecher 1994, 
Pereira et al., 2002). This plant is grown as an either spring or autumn crop, but it is sensitive to cold and dry condition especially at early stages. Worldwide, water shortages and drought stress is among the most important abiotic stresses limiting crop production (Lemoine et al., 2010). Due to the uniformity of the soil environment and lack of environmental control in the field, laboratory investigations have a special importance to assess the tolerance of plants against drought stress. To create an artificial environment controlling water potential, substances with high molecular weight are used. Mannitol, as a high molecular weight substance, owing to inducing a condition with osmotic solution identical to natural environment, is often used to control water potential in drought stress studies and controlled environments (Guo et al., 2011).

Drought stress increases the accumulation of reactive oxygen species (ROS) such as hydrogen peroxide $\left(\mathrm{H}_{2} \mathrm{O}_{2}\right)$, superoxide radicals $\left(\mathrm{O}_{2}^{-}\right)$, and hydroxyl $\left(\mathrm{OH}^{-}\right)$leading to oxidative stress (Saed-Moucheshi et al., 2014b). Production of reactive oxygen species causes lipid peroxidation along with protein and nucleic acid degradation. Plants are able to reduce the damaging effect of reactive oxygen species by different mechanisms. One of these mechanisms is involved in enzymatic or nonenzymatic antioxidant defense system. Antioxidant enzymes such as catalase (CAT), superoxide dismutase (SOD), and peroxidase (POX) are involved in detoxifying oxygen free radicals in plants' cells (Saed-Moucheshi et al., 2014a). In response to increased production of reactive oxygen species, the capacity of antioxidant defense systems and the activity of antioxidant enzymes are increased. The first enzymatic barrier against produced oxidants is SOD, which converts $\mathrm{O}_{2}{ }^{-}$to $\mathrm{H}_{2} \mathrm{O}_{2}$, a molecule with relatively lower radical activity. $\mathrm{H}_{2} \mathrm{O}_{2}$ could be detoxified into water and oxygen by the CAT and ascorbate peroxidase (APX) enzymes. Peroxidase has an important role in detoxification of $\mathrm{H}_{2} \mathrm{O}_{2}$ which can swept away this compound by using ascorbic acid as an electron donor for the reduction of $\mathrm{H}_{2} \mathrm{O}_{2}$ to $\mathrm{H}_{2} \mathrm{O}$ (Saed-Moucheshi et al., 2014b). During this reaction, ascorbic acid is transformed to monodehydroascorbate. POX are glycoproteins that are able to use phenols similar to hydrogen donors to cope with high content of oxygen free radicals. Furthermore, involvement of POX in plant development processes, lignin production, ethylene biosynthesis, plant defenses against stresses, and wound restoration have been proved (Hossain et al., 2015). Changes in the activity of antioxidant enzymes under $\mathrm{NaCl}$ stress (Tian et al., 2016) and heat stress (Lin et al., 2010) have been also reported in broccoli sprouts. Additionally, damages to the proteins along with accumulation of some free amino acids such as proline in order to maintain osmotic pressure leading to regulate protein synthesis in cells has been observed under abiotic stresses (Osakabe et al., 2014). Some researchers claimed that the reduction in synthesis of some types of proteins is attributed to decrease in their polysome numbers (Han and Wagner, 2014, Suzuki et al., 2014). In addition to changes in the content of proteins, lipids are affected by induced abiotic stresses. Membrane lipid peroxidation normally results in production of some aldehyde namely malondialdehyde (MDA) which is a quick responsive compound to oxidative stress (Berger et al., 2016). Furthermore, ethylene and salicylic acid are two phytohormones composed of polyphenols which are important in stress responses of the plants which have direct, indirect, and cascading effects on some vital activities of the plants such as photosynthesis and plant developments under stress conditions (Gupta and Huang, 2014). 
The current study tends to find out about the responses of the different genotypes of the broccoli to water shortage stress caused by mannitol under laboratory and controlled environment through considering different morphological, biochemical, and polyphenols parameters. In addition, to screen the most useful $F_{1}$ hybrids for being used in plant breeding and human nutritional programs. In addition, evaluate the relationship between measured parameters by using advanced and multivariate statistical techniques in order to help breeders to use them as screening criteria for indirect selection of genotypes with higher nutritional qualities.

\section{Materials and methods}

\section{Experimental procedure}

Seeds of the six $F_{1}$ broccoli genotypes (Castle Dome, Green Magic, Heraklion, Marathon, Matsuri and Sacora) prepared from PS America, Inc and TOKITA SEED CO. LTD (Japan) companies were used in a factorial experiment with two factors (Mannitol levels and genotypes) on the bases of completely randomized design (CRD) with three replication and in each replication with 10 petri dishes. The seeds were soaked in ethanol $70 \%$ for 2 minutes and then were in undated with Bleach (sodium hypochlorite) $20 \%$ for 10 minutes; after that, the seeds were washed 4 times with deionized water. Five seeds were transferred to each sterile petri dish containing wet filter papers. The periods of 16 hours of light and 8 hours of darkness in a growth chamber at $23{ }^{\circ} \mathrm{C}$ (day)/ $20{ }^{\circ} \mathrm{C}$ (night) temperature with average humidity of $70 \%$ were applied and continued during the experiment. The five-day small sprouts of broccoli were treated with 88 and $176 \mathrm{mM}$ mannitol for 48 hours (two days). Therefore, the samples were taken from the seven days old sprout to assess the biochemical and growth-related traits.

\section{Measurements of the traits}

Shoot length, root length, and dry weight were measured as growth related traits. Shoot and root lengths were measured using a precise measure on the bases of centimeter. For measurement dry weight, fresh shoots were placed in aluminum foils and kept in an oven for $48 \mathrm{~h}$ under $70{ }^{\circ} \mathrm{C}$, and then were weighed with a precise scale on the bases of gram. The mean of the five used sprout in each petri dish was used for each replication.

Total protein content was measured by the method of Bradford (1976) at $595 \mathrm{~nm}$ spectrophotometric wave length. MDA and $\mathrm{H}_{2} \mathrm{O}_{2}$ contents were estimated by the methods of Heath and Packer (1968) and Velikova et al. (2000), respectively. Enzymatic antioxidants consist of SOD, APX, POX, and CAT were measured using the methods of Giannopolitis and Ries (1977), Nakano and Asada (1981), Dazy et al., (2008), and Aebi (1984), respectively. The methods described by Ainsworth and Gillespie (2007), Oomah and Mazza (1996), and Yuan et al. (2009) were used for the measurement of phenol, flavonoid, and anthocyanin contents in broccoli sprouts, respectively. DPPH as the total activities of the sprout to scavenge reactive oxygen species were estimated using Brand-Williams et al. (1995) method. Using HPLC standard, sulforaphane content in the broccoli sprout was measured for each variety under each treatment after determining the peaks based on the method of Brader et al. (2006). 


\section{Statistical analyses}

Data were analysed by one-way ANOVA in SAS 9.3, followed by Duncan'smultiple range test (DMRT) comparison test. PROC CORR of SAS 9.3 was used for assess Pearson's correlation coefficient between all pairs of measured traits. The graphs for mean comparison were drawn by Excel 2016 software. Multivariate analyses namely principal component analysis (PCA) and biplot graph were assessed by Minitab v. 16.

\section{Results}

\section{Analysis of variance and mean comparison}

Table 1 is presenting the results of analysis of variance for all measured traits. The main effect of genotype was significant $(\mathrm{p}<0.01)$ for all traits. In addition, the effect of mannitol showed significant effect on all measured traits $(p<0.01)$ except for root length. The interaction between genotypes and mannitol was significant for all parameters. Since the interaction effect between genotypes and mannitol showed high significant in the ANOVA table.

The results of multiple mean comparison based on Duncan's multiple range test subjected to growth related traits, shoot length and root length, along with some polyphenols consist of total phenol content, flavonoid content, and anthocyanin content are prepared in Table 2. In all genotypes, control treatment showed higher shoot length than two other levels of mannitol application. In Heraklion, Marathon, Green Magic, and Matsuri genotypes, application of $176 \mathrm{mM}$ mannitol resulted in the lowest shoot length, but in other genotypes application of $88 \mathrm{mM}$ mannitol obtained the lowest shoot length with no significant difference from $176 \mathrm{mM}$. Castle Dome and Matsuri genotypes showed higher shoot length in comparison with other ones. The highest shoot length was achieved in Matsuri genotype under no application of mannitol $(6.87 \mathrm{~cm})$, while the lowest shoot length was obtained in Sacora under the application of $88 \mathrm{mM}$ mannitol $(3.33 \mathrm{~cm})$. Similar to the shoot length, the response of different genotypes to different levels of mannitol were different for root length. In Sacora, Heraklion and Matsuri genotypes, the control treatment showed higher root length than the two other levels of mannitol application. On the contrary, in Marathon, Green magic, and Castle Dome genotypes no application of mannitol resulted in lower root length in compare to other levels. The highest and the lowest root length were obtained in Marathon genotype under the application of $176 \mathrm{mM}$ mannitol $(13.53 \mathrm{~cm})$ and Sacora genotypes under 176 $\mathrm{mM}$ mannitol $(6.83 \mathrm{~cm})$, respectively. Response of dry weight of different broccoli sprouts to different levels of mannitol application is depicted in Table 2. Dry weight of all $F_{1}$ hybrids were reduced by application of mannitol in compare to control. Excluding the Castle Dome genotype, higher level of mannitol application (176 $\mathrm{mM}$ ) resulted in lower dry weight. Matsuri and Castle Dome genotypes showed higher dry weigh than the other genotypes.

Highest total phenol content was obtained in Marathon genotype under $176 \mathrm{mM}$ $\left(112.29 \mathrm{mgg}^{-1} \mathrm{FW}\right)$ while the lowest content was obtained in Sacora genotype under no application of mannitol $\left(39.52 \mathrm{mg} \mathrm{g}^{-1} \mathrm{FW}\right)$. Flavonoid of all genotypes except for Marathon reached a higher content with application of higher mannitol concentration. The highest flavonoid content (123.4 $\left.\mathrm{mgg}^{-1} \mathrm{FW}\right)$ was achieved in Heraklion under highest concentration of mannitol $(176 \mathrm{mM})$, while the lowest 
content achieved in Matsuri under no application of mannitol (28.73 $\left.\mathrm{mg} \mathrm{g}^{-1} \mathrm{FW}\right)$. Anthocyanin content of genotype Matsuri decreased in response to higher application of mannitol. However, the response of all other genotypes to higher concentration of mannitol was to increase the anthocyanin content. Marathon under application of $176 \mathrm{mM}$ mannitol obtained the highest anthocyanin content $(0.623$ $\mathrm{mgg}^{-1} \mathrm{FW}$ ) among all treated plots, while castle Dome genotype under no application of mannitol showed the lowest anthocyanin content $\left(0.14 \mathrm{mgg}^{-1} \mathrm{FW}\right)$.

MDA and $\mathrm{H}_{2} \mathrm{O}_{2}$ as two important markers indicating the severity of the stresses in plant were measured in all genotypes under all mannitol treatments, which their results are presented in Table 2. In all genotypes, higher mannitol content resulted in higher content of both MDA and $\mathrm{H}_{2} \mathrm{O}_{2}$. Heraklion and Marathon showed highest ratio for increase in the content of MDA in response to mannitol application. Similarly, Marathon and Green Magic genotypes showed the highest difference between control and mannitol application among all genotypes regarding $\mathrm{H}_{2} \mathrm{O}_{2}$.

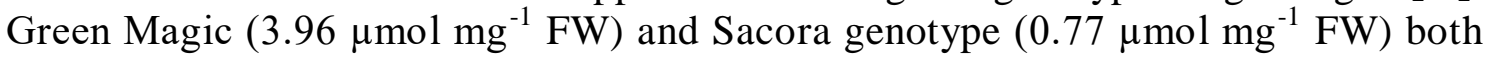
under $176 \mathrm{mM}$ mannitol application resulted in the highest content of MDA and $\mathrm{H}_{2} \mathrm{O}_{2}$, respectively. In contrast, the lowest content of both MDA and $\mathrm{H}_{2} \mathrm{O}_{2}$ were observed in Castle Dome genotype under no application of mannitol.

SOD, CAT, POX, and APX as the enzymatic ROS scavengers were measured using spectrophotometric tool. The response of all enzymatic antioxidants to higher concentration of mannitol were to increase their activities in all genotypes. The rate of increase in Matsuri genotype regarding SOD was higher than other genotypes (104 and 166\% increase in 88 and $176 \mathrm{mM}$ mannitol in relation to control). Similar results regarding other measured enzymatic antioxidant were observed for this variety (Table 2). Excluding CAT, Marathon genotype showed the highest enzymatic activities among all genotypes under $176 \mathrm{mM}$ mannitol application. Heraklion genotype under highest application mannitol showed the highest activity for CAT. The lowest activity of SOD, CAT, POX, and APX were observed in Sacora, Matsuri, Green Magic and Sacora under control treatment, respectively. The activity of DPPH which is an abbreviation for 2, 2-diphenyl-1-picrylhydrazyl was also measured as a free radical scavenger (Table 2). In genotype Marathon, the antioxidant activity by DPPH under no application of mannitol showed a high significant difference with application of $88 \mathrm{mM}$ mannitol, but application of 176 $\mathrm{mM}$ showed a lower significant activity in compare to control. Similarly, in all other genotypes, the higher concentration of mannitol resulted in higher activity of DPPH. The highest activity of DPPH achieved in Heraklion genotype under the application of $176 \mathrm{mM}$ mannitol $\left(85.46 \mathrm{mmol} \mathrm{kg}{ }^{-1}\right)$, but the lowest activity was resulted from control treatment in var. Matsuri (64.71 $\left.\mathrm{mmol} \mathrm{kg}^{-1}\right)$.

Sulfurphane as an important compound in broccoli was also measured in all genotypes under all treatments, which its results are presented in Fig. 1. The response of all genotypes to application of mannitol were to increase the content of sulforaphane but the rate of increase in some genotypes consist of Sacora and Castle Dome were low. The rate of increase in the content of sulforaphane in Masuri and Heraklion was higher in response to increase the concentration of mannitol with a high positive trend. Marathon and Green Magic showed a great increase from no application to $88 \mathrm{mM}$ application of mannitol, but the difference between 88 and 176 $\mathrm{mM}$ mannitol remained insignificant. Under all mannitol levels and in total, the content of sulforaphane was highest in Marathonin comparison to other genotypes. 


$$
-2048 \text { - }
$$

Table 1. Analysis of variance for measured traits in six F1 hybrids broccoli under mannitol treatment

\begin{tabular}{|c|c|c|c|c|c|c|c|c|c|c|c|c|c|c|c|}
\hline \multirow{2}{*}{ Source } & \multirow{2}{*}{ DF } & \multicolumn{14}{|c|}{ Mean Square } \\
\hline & & DW & $\begin{array}{l}\text { Shoot } \\
\text { length }\end{array}$ & $\begin{array}{c}\text { Root } \\
\text { length }\end{array}$ & $\begin{array}{c}\text { Sulfo- } \\
\text { raphane }\end{array}$ & MDA & $\mathbf{H}_{2} \mathbf{O}_{2}$ & Phenol & Flavonoid & $\begin{array}{l}\text { Antho- } \\
\text { cyanin }\end{array}$ & SOD & CAT & POX & APX & DPPH \\
\hline Variety & 5 & $0.17 * *$ & $5.64 * *$ & $40.3 * *$ & $46.58 * *$ & $4.18 * *$ & $0.32 * *$ & $3465.78 * *$ & $1314.91 * *$ & $0.24 * *$ & $5910.37 * *$ & $1220.73 * *$ & $764.16^{* *}$ & $4187.53^{* *}$ & $57.45 * *$ \\
\hline Mannitol & 2 & $0.06^{* *}$ & $11.42 * *$ & $1.05 \mathrm{~ns}$ & $139.3^{* *}$ & $0.3^{* *}$ & $0.07 * *$ & $1338.42 * *$ & $1985.89 * *$ & $0.05^{* *}$ & $5891.76^{* *}$ & $2493.38 * *$ & $999.84 * *$ & $11596.67 * *$ & $169.84^{* *}$ \\
\hline Interaction & 10 & $0.02 * *$ & $1.56^{* *}$ & $2.97 * *$ & $15.17 * *$ & $0.6^{* *}$ & $0.14 * *$ & $106.31 * *$ & $999.98 * *$ & $0.005^{* *}$ & $446.94 * *$ & $135.99 * *$ & $89.54 * *$ & $363.52 * *$ & $58.15 * *$ \\
\hline Error & 36 & 0.001 & 0.05 & 0.33 & 0.27 & 0.001 & 0.0004 & 4.68 & 2.03 & 0.0003 & 2.46 & 1.04 & 0.47 & 0.86 & 0.57 \\
\hline \multicolumn{2}{|c|}{$\begin{array}{l}\text { Coefficient of } \\
\text { Variation }\end{array}$} & 3.51 & 4.59 & 5.71 & 6.47 & 1.23 & 6.46 & 3.7 & 2.06 & 4.16 & 2.39 & 2.98 & 3.61 & 1.96 & 0.92 \\
\hline
\end{tabular}

**, *, and ns: significant at $0.01,0.05$ level, and non-significant at 0.05 level.

DW: Dry wight, MDA: Malondialdehyde, H2O2: hydrogen peroxide, SOD: Super Oxide Dismutase, CAT:Catalase, POX: Guaiacul Peroxidase,

APX:Ascorbic Peroxidase, DPPH:1,1-diphenyl-2-picrylhydrazyl

Table 2. Multiple mean comparison of measured traits for interaction between broccoli varieties and mannitol treatment

\begin{tabular}{|c|c|c|c|c|c|c|c|c|c|c|c|c|c|c|}
\hline Variety & $\underset{(\mathrm{mM})}{\text { Mannitol }}$ & $\begin{array}{c}\text { Dry } \\
\text { weight } \\
\text { (g) }\end{array}$ & $\begin{array}{l}\text { Shoot } \\
\text { length } \\
(\mathbf{c m})\end{array}$ & $\begin{array}{l}\text { Root } \\
\text { length } \\
\text { (cm) }\end{array}$ & $\begin{array}{c}\text { Phenol } \\
(\mathrm{mg} / \mathrm{g} \text { FW) }\end{array}$ & $\begin{array}{c}\text { Flavonoid } \\
(\mathrm{mg} / \mathrm{g} \text { FW })\end{array}$ & $\begin{array}{c}\text { Antho- } \\
\text { cyanin } \\
(\mathrm{mg} / \mathrm{g} \text { FW) }\end{array}$ & $\begin{array}{c}\text { MDA } \\
\left(\mu \mathrm{mol}^{-1}\right. \\
\mathbf{m g}^{-1} \\
\text { FW }) \\
\end{array}$ & $\begin{array}{c}\mathrm{H}_{2} \mathrm{O}_{2} \\
\left(\mu \mathrm{mol}^{-1}\right. \\
\mathrm{mg}^{-1} \\
\mathrm{FW}) \\
\end{array}$ & $\begin{array}{c}\text { SOD } \\
(\mu \mathrm{mol} \\
\mathrm{mm}^{-1} \\
\left.\mathrm{mg}^{-1} \mathbf{p}\right)\end{array}$ & $\begin{array}{c}\text { CAT } \\
(\mu \mathrm{mol} \\
\mathbf{m m}^{-1} \\
\left.\mathbf{m g}^{-1} \mathbf{p}\right) \\
\end{array}$ & $\begin{array}{c}\text { POX } \\
(\mu \mathrm{mol} \\
\mathbf{m m}^{-1} \\
\left.\mathrm{mg}^{-1} \mathbf{p}\right) \\
\end{array}$ & $\begin{array}{c}\text { APX } \\
(\mu \mathrm{mol} \\
\mathbf{m m}^{-1} \\
\left.\mathbf{m g}^{-1} \mathbf{p}\right) \\
\end{array}$ & $\begin{array}{c}\text { DPPH } \\
\left(\mathrm{mmol} \mathrm{kg}^{-1}\right)\end{array}$ \\
\hline \multirow{3}{*}{ Sacora } & 0 & $0.36 \mathrm{H}$ & $6.367 \mathrm{~B}$ & $7.833 \mathrm{I}$ & $39.524 \mathrm{~L}$ & $63.667 \mathrm{G}$ & $0.451 \mathrm{E}$ & $2.551 \mathrm{H}$ & $0.23 \mathrm{~J} 1$ & $24.011 \mathrm{~L}$ & $26.472 \mathrm{I}$ & $10.939 \mathrm{~J}$ & $14.976 \mathrm{~N}$ & $82.323 \mathrm{~F}$ \\
\hline & 88 & $0.32 \mathrm{I}$ & $3.333 \mathrm{~K}$ & 7.667IJ & $47.476 \mathrm{HIJ}$ & $83.067 \mathrm{C}$ & $0.505 \mathrm{D}$ & $2.722 \mathrm{G}$ & $0.35 \mathrm{G}$ & $37.553 \mathrm{~J}$ & $32.672 \mathrm{FG}$ & $11.222 \mathrm{~J}$ & $40.422 \mathrm{I}$ & 84.091BCDE \\
\hline & 176 & $0.314 \mathrm{I}$ & 4.467FG & $6.833 \mathrm{~J}$ & 66.333D & $83.933 \mathrm{C}$ & $0.582 \mathrm{~B}$ & $2.935 \mathrm{~F}$ & $0.77 \mathrm{~A}$ & $31.146 \mathrm{~K}$ & $35.637 \mathrm{E}$ & $13.043 \mathrm{I}$ & $55.255 \mathrm{G}$ & $84.495 \mathrm{ABC}$ \\
\hline \multirow{3}{*}{ Heraklion } & 0 & $0.41 \mathrm{G}$ & $5.567 \mathrm{CD}$ & $13.033 \mathrm{AB}$ & $45.238 \mathrm{JK}$ & $51.733 \mathrm{~J}$ & $0.487 \mathrm{D}$ & $2.373 \mathrm{I}$ & $0.2 \mathrm{H}$ & $66.522 \mathrm{~F}$ & $33.413 \mathrm{~F}$ & $26.287 \mathrm{E}$ & $27.013 \mathrm{~K}$ & $82.02 \mathrm{FG}$ \\
\hline & 88 & $0.344 \mathrm{H}$ & $4.7 \mathrm{~F}$ & 11.1D & $52.317 \mathrm{EFG}$ & $72 \mathrm{E}$ & $0.534 \mathrm{C}$ & $3.223 \mathrm{D}$ & $0.44 \mathrm{~F}$ & $76.005 \mathrm{E}$ & $48.789 \mathrm{C}$ & $30.866 \mathrm{D}$ & $71.515 \mathrm{E}$ & $85.152 \mathrm{AB}$ \\
\hline & 176 & $0.35 \mathrm{H}$ & $3.567 \mathrm{JK}$ & $12.5 \mathrm{BC}$ & $51.619 \mathrm{EFG}$ & $123.4 \mathrm{~A}$ & $0.543 \mathrm{C}$ & $3.869 \mathrm{~B}$ & $0.52 \mathrm{E}$ & $82.162 \mathrm{D}$ & $64.764 \mathrm{~A}$ & $37.062 \mathrm{~B}$ & $95.819 \mathrm{~B}$ & $85.455 \mathrm{~A}$ \\
\hline
\end{tabular}




\begin{tabular}{|c|c|c|c|c|c|c|c|c|c|c|c|c|c|c|}
\hline \multirow{3}{*}{ Marathon } & 0 & $0.355 \mathrm{H}$ & $4.3 \mathrm{GH}$ & $12.067 \mathrm{C}$ & $85.143 \mathrm{C}$ & $58.333 \mathrm{HI}$ & $0.423 \mathrm{E}$ & $2.049 \mathrm{~K}$ & $0.16 \mathrm{G}$ & $82.394 \mathrm{D}$ & $32.415 \mathrm{FG}$ & $15.002 \mathrm{H}$ & $45.985 \mathrm{H}$ & $75.96 \mathrm{H}$ \\
\hline & 88 & $0.314 \mathrm{I}$ & $4.033 \mathrm{HI}$ & $13.167 \mathrm{AB}$ & 97.952B & $72.133 \mathrm{E}$ & $0.543 \mathrm{C}$ & $2.223 \mathrm{~J}$ & $0.3 \mathrm{~J}$ & 101.629B & $45.854 \mathrm{D}$ & $26.929 \mathrm{E}$ & $84.076 \mathrm{C}$ & $85.404 \mathrm{~A}$ \\
\hline & 176 & $0.293 \mathrm{IJ}$ & $3.8 \mathrm{IJ}$ & $13.533 \mathrm{~A}$ & $112.286 \mathrm{~A}$ & $91.133 \mathrm{~B}$ & $0.623 \mathrm{~A}$ & $3.055 \mathrm{E}$ & $0.53 \mathrm{D}$ & $126.464 \mathrm{~A}$ & $55.502 \mathrm{~B}$ & 42.997A & $110.646 \mathrm{~A}$ & 83.737CDE \\
\hline \multirow{3}{*}{ Green Magic } & 0 & $0.46 \mathrm{~F}$ & $5.1 \mathrm{E}$ & $8.933 \mathrm{GH}$ & 45.667IJK & $57.086 \mathrm{I}$ & $0.369 \mathrm{~F}$ & $3.21 \mathrm{D}$ & $0.18 \mathrm{HI}$ & $55.538 \mathrm{H}$ & $21.558 \mathrm{~J}$ & $5.477 \mathrm{M}$ & $16.437 \mathrm{~N}$ & $82.273 \mathrm{FG}$ \\
\hline & 88 & $0.39 \mathrm{G}$ & 4.4FGH & $8.5 \mathrm{GHI}$ & $50.571 \mathrm{FGH}$ & $71.467 \mathrm{E}$ & $0.482 \mathrm{D}$ & $3.619 \mathrm{C}$ & $0.64 \mathrm{C}$ & $76.06 \mathrm{E}$ & $31.465 \mathrm{G}$ & $21.666 \mathrm{~F}$ & $23.257 \mathrm{~L}$ & 83.232DEF \\
\hline & 176 & $0.35 \mathrm{H}$ & $3.267 \mathrm{~K}$ & $10.667 \mathrm{DE}$ & $54.952 \mathrm{E}$ & $75.533 \mathrm{D}$ & $0.535 \mathrm{C}$ & $3.958 \mathrm{~A}$ & $0.71 \mathrm{~B}$ & $96.427 \mathrm{C}$ & $44.827 \mathrm{D}$ & $33.826 \mathrm{C}$ & $34.731 \mathrm{~J}$ & 84.394ABCD \\
\hline \multirow{3}{*}{ Matsuri } & 0 & $0.89 \mathrm{~A}$ & $6.867 \mathrm{~A}$ & 9.933FE & $40.952 \mathrm{~L}$ & $28.733 \mathrm{~K}$ & $0.214 \mathrm{G}$ & $1.695 \mathrm{M}$ & $0.09 \mathrm{JK}$ & $18.845 \mathrm{M}$ & $11.419 \mathrm{~L}$ & $5.334 \mathrm{M}$ & $18.729 \mathrm{M}$ & 64.717I \\
\hline & 88 & $0.83 \mathrm{~B}$ & $5.7 \mathrm{C}$ & $8.1 \mathrm{HI}$ & $48.952 \mathrm{GHI}$ & $53.133 \mathrm{~J}$ & $0.195 \mathrm{GH}$ & $1.957 \mathrm{~L}$ & $0.06 \mathrm{KL}$ & $40.68 \mathrm{I}$ & $29.054 \mathrm{H}$ & $11.405 \mathrm{~J}$ & $44.655 \mathrm{H}$ & $82.929 \mathrm{EF}$ \\
\hline & 176 & $0.65 \mathrm{E}$ & $4.333 \mathrm{FGH}$ & 7.667IJ & $67.238 \mathrm{D}$ & $60.333 \mathrm{H}$ & $0.199 \mathrm{GH}$ & $2.051 \mathrm{~K}$ & $0.16 \mathrm{~J}$ & $74.025 \mathrm{E}$ & $55.548 \mathrm{~B}$ & $16.032 \mathrm{GH}$ & 80.11D & 84.293ABCD \\
\hline \multirow{3}{*}{ Castle Dome } & 0 & $0.9 \mathrm{~A}$ & $6.6 \mathrm{AB}$ & $9.333 \mathrm{FG}$ & $42.667 \mathrm{KL}$ & $53 \mathrm{~J}$ & $0.14 \mathrm{I}$ & $1.701 \mathrm{M}$ & $0.01 \mathrm{M}$ & $35.627 \mathrm{~J}$ & $10.782 \mathrm{~L}$ & $6.883 \mathrm{~L}$ & 9.3820 & $81.061 \mathrm{G}$ \\
\hline & 88 & $0.72 \mathrm{D}$ & $5.233 \mathrm{DE}$ & $10.667 \mathrm{DE}$ & $53.524 \mathrm{EF}$ & $81.267 \mathrm{C}$ & $0.179 \mathrm{H}$ & $1.938 \mathrm{~L}$ & $0.05 \mathrm{~L}$ & $59.043 \mathrm{G}$ & $15.741 \mathrm{~K}$ & $9.733 \mathrm{~K}$ & $18.207 \mathrm{M}$ & 83.586CDE \\
\hline & 176 & $0.79 \mathrm{C}$ & $5.933 \mathrm{~B}$ & $10.833 \mathrm{DE}$ & $50.238 \mathrm{FGH}$ & $67.067 \mathrm{~F}$ & $0.219 \mathrm{G}$ & $2.05 \mathrm{~K}$ & $0.15 \mathrm{j}$ & $95.877 \mathrm{C}$ & $20.97 \mathrm{~J}$ & $16.338 \mathrm{G}$ & $60.532 \mathrm{~F}$ & 84.04BCDE \\
\hline
\end{tabular}

Means with the same letter(s) in each column are not significantly different (Duncan 1\%). 


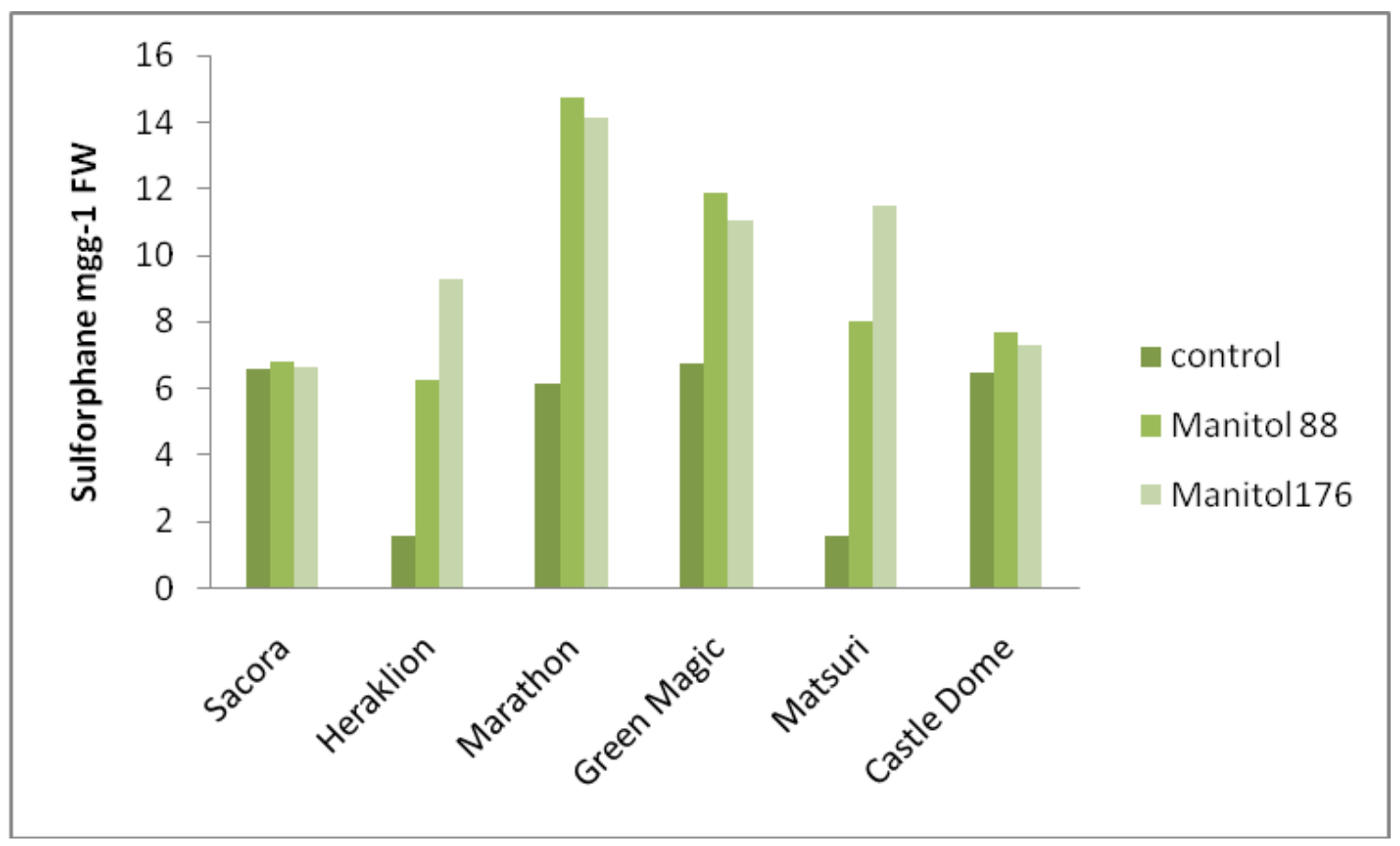

Figure 1. Response of sulfonphan content of different broccoli genotypes to mannitol treatment

\section{Correlation between measured traits}

The pairwise correlations between all pairs of measured traits based on Pearson method is presented in Table 3. Except for shoot length (0.74), dry weight showed negative correlations with all other traits. Similar to dry weight, all pairwise correlations of shoot length, excluding dry weight, were negative. The only insignificant correlation of shoot length was related to root length (-0.19). Root length showed negative correlation with shoot length and dry weight but these correlations were not statistically significant. Excluding dry weight and shoot length, all other correlations of root length were positive, but its correlation only with phenol content (0.5), SOD (0.7), and POX (0.67) were significant. Sulforaphane showed significant correlations with shoot length (-0.61), phenol content (0.65), SOD (0.66), CAT (0.58), POX (0.52), APX (0.58), and DPPH (0.47). The content of sulforaphane showed no significant correlations with flavonoid (0.29) and anthocyanin (0.31). MDA revealed negative significant correlations with dry weight (-0.67) and shoot length (-0.57). Also, the correlations of MDA with $\mathrm{H}_{2} \mathrm{O}_{2}(0.79)$, flavonoid (0.61), anthocyanin (0.61), CAT (-0.54), and POX ($0.51)$ were significant. Similar to MDA, the correlations of $\mathrm{H}_{2} \mathrm{O}_{2}$ with dry weight ($0.66)$ and shoot length (-0.63) were negatively significant. Furthermore, $\mathrm{H}_{2} \mathrm{O}_{2}$ showed significant correlations with MDA (0.79), flavonoid (0.59), anthocyanin (0.63), SOD (0.43), CAT (-0.64), POX (-0.47). Phenol content showed significant positive correlations with all enzymatic antioxidant. The correlation of flavonoid with CAT was positively significant (0.51), but its correlations with other enzymatic antioxidant were not significant. Anthocyanin showed positive significant correlations with CAT (0.63) and POX (0.69). All internal correlations of enzymatic antioxidants were positively significant. DPPH as a stable compound with antioxidant ability showed positive significant correlation with SOD (0.48), Cat (0.53) and POX (0.49) but its correlations with APX were not significant. 


$$
-2051 \text { - }
$$

Table 3. Pearson correlation coefficients for all pairs of the traits measured in different varieties of broccoli under mannitol treatment

\begin{tabular}{|c|c|c|c|c|c|c|c|c|c|c|c|c|c|c|}
\hline & DW & SL & $\mathbf{R L}$ & DPPH & MDA & $\mathrm{H}_{2} \mathrm{O}_{2}$ & Phenol & Flavonoid & $\begin{array}{l}\text { Antho- } \\
\text { cyanin }\end{array}$ & SOD & CAT & POX & APX & $\begin{array}{c}\text { Sulfo- } \\
\text { raphane }\end{array}$ \\
\hline DW & 1 & & & & & & & & & & & & & \\
\hline SL & $0.74 * *$ & 1 & & & & & & & & & & & & \\
\hline RL & $\stackrel{-}{0.23 \mathrm{~ns}}$ & $\begin{array}{c}- \\
0.19 \mathrm{~ns}\end{array}$ & 1 & & & & & & & & & & & \\
\hline DPPH & $-0.52 *$ & $-0.53^{*}$ & $\begin{array}{c}0.41 \mathrm{n} \\
\mathrm{s}\end{array}$ & 1 & & & & & & & & & & \\
\hline MDA & $\begin{array}{c}- \\
0.67 * *\end{array}$ & $-0.57 *$ & $\begin{array}{c}0.04 n \\
s\end{array}$ & $0.22 \mathrm{~ns}$ & 1 & & & & & & & & & \\
\hline $\mathrm{H}_{2} \mathrm{O}_{2}$ & $\stackrel{-}{-}$ & ${ }^{-}-3^{* *}$ & $\begin{array}{c}0.06 \mathrm{n} \\
\mathrm{s}\end{array}$ & $\begin{array}{c}- \\
0.46 \mathrm{~ns}\end{array}$ & $0.79 * *$ & 1 & & & & & & & & \\
\hline Phenol & $0 . \overline{42 \mathrm{~ns}}$ & $-0.5^{*}$ & $0.5^{*}$ & $0.16 \mathrm{~ns}$ & $\begin{array}{c}- \\
0.12 \mathrm{~ns}\end{array}$ & $0.18 \mathrm{~ns}$ & 1 & & & & & & & \\
\hline Flavonoid & $-0.53^{*}$ & $0.65^{* *}$ & $\begin{array}{c}0.17 \mathrm{n} \\
\mathrm{s}\end{array}$ & $0.35 \mathrm{~ns}$ & $0.61 * *$ & $0.59 * *$ & $0.16 \mathrm{~ns}$ & 1 & & & & & & \\
\hline Anthocyanin & $\begin{array}{c}- \\
0.95^{* *}\end{array}$ & $\begin{array}{c}- \\
0.68 * *\end{array}$ & $\begin{array}{c}0.31 \mathrm{n} \\
\mathrm{s}\end{array}$ & $0.34 \mathrm{~ns}$ & $0.61 * *$ & $0.63 * *$ & $0.42 \mathrm{~ns}$ & $0.44 \mathrm{~ns}$ & 1 & & & & & \\
\hline SOD & $\stackrel{-}{0.38 \mathrm{~ns}}$ & $-0.52 *$ & $0.7 * *$ & $0.48^{*}$ & $\begin{array}{c}- \\
0.22 \mathrm{~ns}\end{array}$ & $-0.43 *$ & $0.69 * *$ & $0.26 \mathrm{~ns}$ & $0.39 \mathrm{~ns}$ & 1 & & & & \\
\hline CAT & $0.63^{-} *$ & $0.75^{* *}$ & $\begin{array}{c}0.34 n \\
s\end{array}$ & $0.53 *$ & $-0.54 *$ & $0.64 * *$ & $0.52 *$ & $0.51 *$ & $0.63 * *$ & $0.6^{* *}$ & 1 & & & \\
\hline POX & $-0.58^{*}$ & $-0.6 * *$ & $0.67 * *$ & $0.49 *$ & $-0.51^{*}$ & $-0.47 *$ & $0.51^{*}$ & $0.38 \mathrm{~ns}$ & $0.69 * *$ & $0.79 * *$ & $0.82 * *$ & 1 & & \\
\hline APX & - & $-0.54 *$ & $\begin{array}{c}0.44 n \\
s\end{array}$ & $0.3 \mathrm{~ns}$ & $0 . \overline{24 \mathrm{~ns}}$ & $0 . \overline{11 \mathrm{~ns}}$ & $0.7 * *$ & $0.38 \mathrm{~ns}$ & $0.44 \mathrm{~ns}$ & $0.67 * *$ & $0.84 * *$ & $0.72 * *$ & 1 & \\
\hline Sulforaphane & $0.34 \mathrm{~ns}$ & $0.61^{-} * *$ & $\begin{array}{c}0.16 n \\
s\end{array}$ & $0.47 *$ & $0.18 \mathrm{~ns}$ & $\begin{array}{c}- \\
0.19 \mathrm{~ns}\end{array}$ & $0.65^{* *}$ & $0.29 \mathrm{~ns}$ & $0.31 \mathrm{~ns}$ & $0.66^{* *}$ & $0.58 *$ & $0.52 *$ & $0.58 *$ & 1 \\
\hline
\end{tabular}

$* *, *$, and ns: significant at $0.01,0.05$ level, and not-significant at 0.05 . 
To achieve more sophisticated results without the effect of other correlated variables on sulforaphane, the direct effect of all variables by holding all the effect of all other variable fixed using path coefficient analysis were implemented (Table 4). The path analysis showed that dry weight, $\mathrm{H}_{2} \mathrm{O}_{2}$ content, and then phenol content were the most influential variables positively affecting the content of sulforaphane, respectively. On the other hand, shoot length showed a negative coefficient in the path analysis. The coefficient of all other variables in the path analysis were negligible.

\section{Principal component}

Principal component analysis was carried out to assess the inter relationship among 14 measured traits in this study. The results showed that the first two PCs accounted for about 81 percent of total variability. Therefore, the biplot for the first two PCs in which both genotypes and parameters can be depicted in a same plot was prepared (Table 5). Dry weight and shoot length showed negative association with all other traits because they placed in a revers proximity to all other traits in the two dimensional graph of first two components. Matsuri and Castle Dome as two close genotypes were placed in the area near to the dry weigh and shoot length. Root length, sulforaphane content, phenol content, APX, SOD, POX, and CAT showed acute angles one another and also had positive high coefficients with both first and second components. Marathon was fixed solely in adjacent to these traits nearer to sulforaphane than all other genotypes. $\mathrm{H}_{2} \mathrm{O}_{2}$, MDA, DPPH, flavonoid content, and anthocyanin content showed positive high coefficients with first component but negative high coefficient regarding the second component. Genotypes Heraklion and Green Magic placed in near to these parameters, while Green Magic showed closest distance with DPPH and Heraklion with flavonoid content. Genotype Sacora showed a revers trend to all other genotypes considered in this study. Sacora obtained negative high coefficient with both components and was solely placed in a separate group (Fig. 2).

Table 4. Direct effect of each variable through sulforaphane content

\begin{tabular}{c|c}
\hline Variable & Coefficient \\
\hline DW & 0.850992 \\
Shoot length & -0.52174 \\
Root length & 0.041577 \\
$\mathrm{MDA}$ & 0.266981 \\
$\mathrm{H}_{2} \mathrm{O}_{2}$ & 0.848199 \\
Phenol & 0.771737 \\
Flavonoeid & -0.04394 \\
antocyanin & -0.16695 \\
SOD & -0.03846 \\
CAT & -0.32892 \\
POD & -0.37341 \\
APX & 0.407253 \\
DPPH & 0.458507 \\
\hline
\end{tabular}


Table 5. Proportion of each principal component and the related score of each trait

\begin{tabular}{c|c|c}
\hline & PC1 & PC2 \\
\hline Eigenvalue & 7.9454 & 3.3633 \\
Proportion & 0.567529 & 0.240236 \\
Cumulative & 0.567529 & 0.807764 \\
\hline DW & -0.323 & -0.186 \\
Shoot length & -0.333 & -0.062 \\
Root length & 0.228 & -0.322 \\
sulforaphane & 0.172 & -0.266 \\
MDA & 0.242 & 0.351 \\
$\mathrm{H}_{2} \mathrm{O}_{2}$ & 0.311 & 0.212 \\
Phenol & 0.204 & -0.404 \\
Flavonoeid & 0.262 & 0.23 \\
Aantocyanin & 0.328 & 0.173 \\
SOD & 0.247 & -0.305 \\
CAT & 0.291 & -0.085 \\
POD & 0.322 & -0.109 \\
APX & 0.215 & -0.364 \\
DPPH & 0.186 & 0.358 \\
\hline
\end{tabular}

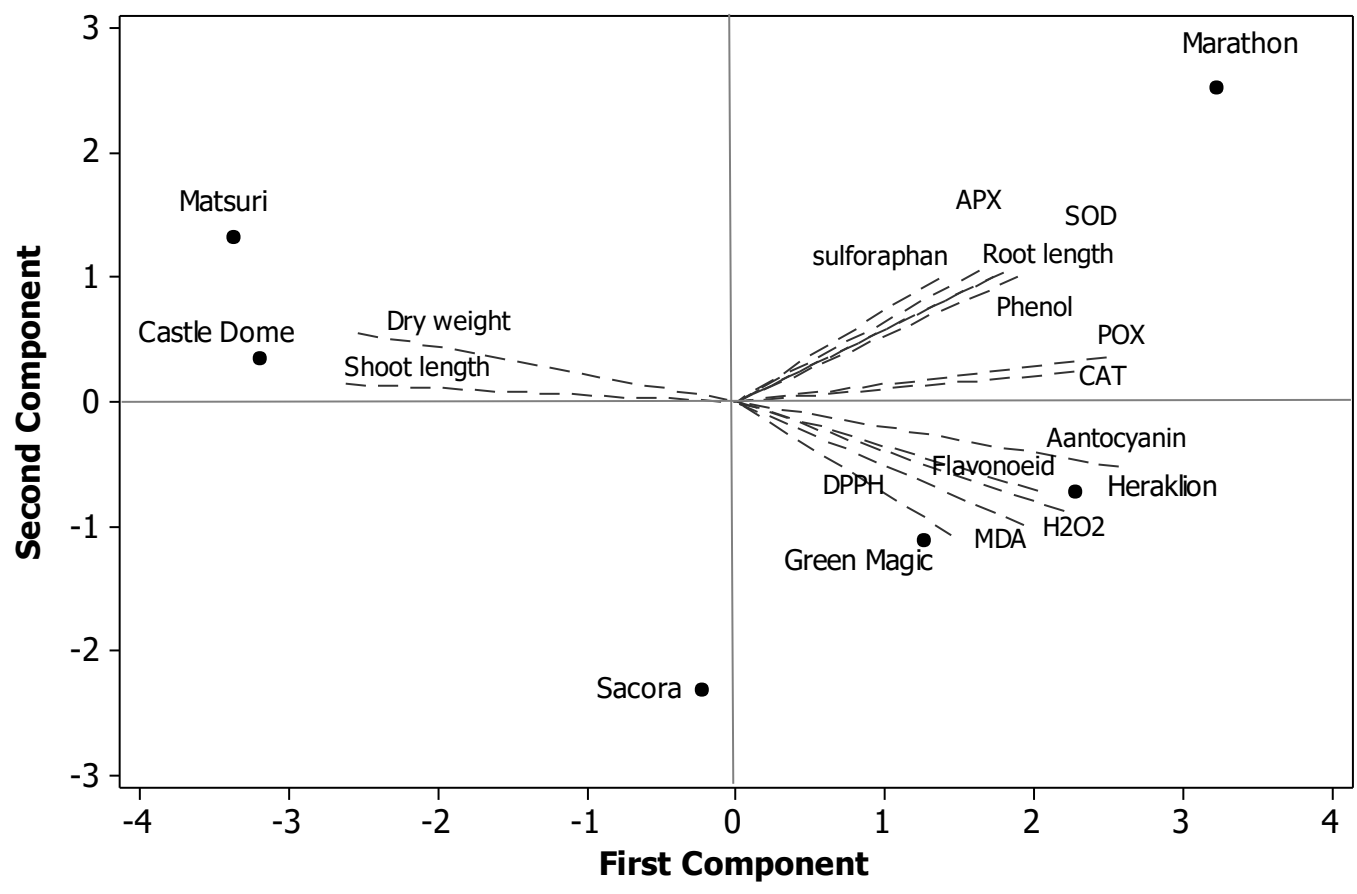

Figure 2. Biplot genotypes and traits using first two components of principal component analysis 


\section{Discussion}

The results of the current study indicated that application of mannitol significantly decreased shoot dry weight and shoot length in all genotypes. Some genotypes had lower decrease rate in comparison with other genotypes regarding shoot length (Marathon genotype) and shoot dry weight (Marathon and Heraklion genotypes), however they might not show the highest values for these traits. Root length response to mannitol application in different genotypes was varied. Most of the genotypes showed increase in root length in response to mannitol application but some genotypes (Sacora and Heraklion) showed decrease. However, the rate of decrease in Heraklion was not significant. The reduction of shoot length and dry weight might be due to the falling off in the rate of photosynthesis under osmotic stress caused by mannitol application as a representative of drought stress. Chaves et al. (2009) reported that under drought stress the closure of stomata occurs to maintain the water content in the plant cells, which directly affects the growth rate of the plant's shoot. Also, the decline in the leaf area and other photosynthetic part of the plant is doubled with the stomatal closure and make photosynthesis rate to be even more reduced (Berger et al., 2016). On the other hand, the reaction of the root to drought stress might be different from what is predictable in shoot. It is well established that the root of the plants is seeking for water storage by increase its length and number of lateral roots and hairy roots (Chaves et al., 2009). The subject of root system architecture (RSA) under drought stress is revealing that under drought stress, the growth of the root can be increased, maintained at determined level, or even decreased; but the rate of growth in the shoot would normally decrease, and so that the overall growth of the root under drought stress would be always higher than the shoot (Uga et al., 2013). Furthermore, it has been stated by some researchers that tolerant genotypes are normally responding to drought in different ways which is one of the most effective way is to enlarge the root growth, while the susceptible genotypes are not able to manage this growth as properly as the tolerant genotypes (De Dorlodot et al., 2007).

In all genotypes, the content of total phenols, flavonoid, and anthocyanin increased. Marathon and Heraklion genotype showed relatively higher content of these three traits than other genotypes, while the Marathon genotype could be stand out as the highest responsive variety. Based on the study of Gawlik-Dziki (2008) phenolic compounds in vegetables, herb, and other edible plants can make them act as reducing agents and respond to oxidant compounds. In this situation, phenolic compounds can be used as hydrogen donators leading to quench the free radicals. Phenolic acids and flavonoids reportedly have shown strong antioxidant properties. They are able to react to radicals and catalyze oxidative reactions resulted in scavenging free radicals and stabilizing them in plants (Saed-Moucheshi et al., 2014b). Under drought stress, the content of free radicals or ROS in plant's cells is arisen which normally causes damages to cell apparatus; therefore, higher content of phenolic compounds indicates higher tolerance of genotype and plants to drought stress. Moreover, it has been reported that antioxidant activity of edible parts of the plants is linked with antimutagenic and anticancer genic properties. Subsequently, genotypes with higher content of natural antioxidants as phenolic compounds can be suggested for being used in nutritional programs and food content of human. In addition to broccoli, the extracts of varies fruits and vegetables have showed higher phenolic compounds in response to drought stress (Gawlik-Dziki, 2008). 
MDA and $\mathrm{H}_{2} \mathrm{O}_{2}$ content are two important markers of oxidative stress induced in plants (Vosough et al., 2015). The lower the content of these two compounds under stress condition, the lower the damages to the plants under this situation (SaedMoucheshi et al., 2014b). Genotypes and cultivars with lower content of MDA and $\mathrm{H}_{2} \mathrm{O}_{2}$ under stress condition would reveal higher tolerant to stress. In this study the content of both MDA and $\mathrm{H}_{2} \mathrm{O}_{2}$ increased under drought stress; however, the response rates of genotypes were varied. Castle Dome showed lower content of these compounds than other genotypes under all mannitol levels. In addition, the difference between mannitol levels in Marathon and Heraklion were relatively lower in compare to other genotypes. Under drought stress, the generation of ROS is increased from the steady level in the plant result in oxidative stress. $\mathrm{H}_{2} \mathrm{O}_{2}$ and other high reactive oxygen compounds can directly react with lipids and other apparatuses of cell membrane leading to lipid peroxidation and higher content of MDA (Hossain et al., 2015).

The activity of all assessed enzymatic antioxidant in this study increased in all genotypes in response to the increase of mannitol levels. Among all genotypes, Marathon showed relatively higher activity for this antioxidant under mannitol applications. In addition, Heraklion showed higher activity of this antioxidant under drought stress. Additionally, DPPH antioxidant activity indicated that the antioxidant activity of broccoli sprout is higher under drought stress condition. Marathon variety showed a high increase in the DPPH antioxidant activity in response to mannitol levels. The induced response of drought stress is to heighten the generation of ROS, which in turn induces the activity of antioxidant compounds and specially the enzymatic ones. In varies studies, higher activity for enzymatic antioxidant under stress condition indicates higher ability of the plants in response to stress condition (Gupta and Huang, 2014; Hossain et al., 2015; Nakano and Asada, 1981). Based on this viewpoint, genotypes with higher activity of enzymatic antioxidant can be considered as higher tolerant genotypes to drought stress. Changes in the activity of antioxidant enzymes under various environmental stresses have been also reported (Baxter et al., 2014). In addition to phenolic compounds and enzymatic antioxidant, DPPH which itself is a radical compound normally acts as a scavenger for other dangerous radicals and therefore its content is a marker for higher antioxidant activity in plants.

The response of all genotypes to application of mannitol were to increase the content of sulforaphane but the rate of increase in some genotypes consist of Sacora and Castle Dome genotypes were low. Marathon and Green magic showed a great increase from zero to $88 \mathrm{mM}$ application of mannitol, but the difference between 88 and $176 \mathrm{mM}$ mannitol remained non-significant. Under all mannitol levels and in total, the content of sulforaphane was highest in Marathon in comparison to other genotypes. Owing to the anticarcinogenic and anticancer functions, sulforaphane is an important compound in broccoli sprout. Since sulforaphane is a naturally available inducer of phase II enzymes in human and animal bodies that is able to detoxify cancer-causing chemicals, it would be appropriate to use such foods and plants part as broccoli, which are containing this compound to use its anti-cancerous properties. Additionally, sulforaphane has a cytoprotective effect against oxidative stress (Gu et al., 2011).

Considering the relationship among measured parameters, it is turned out that the shoot dry weight along with shoot length showed negative correlation with other measured traits. There were positive correlations among sulforaphane content, root length, total phenol content, SOD, APX, CAT, and POX. In addition, there were significant correlations among flavonoid content, anthocyanin content, MDA, $\mathrm{H}_{2} \mathrm{O}_{2}$. Gu et al. 
(2011) investigated the effect of sucrose on the sulforaphane content in broccoli sprouts and stated that there was a significant correlation between glucosinolate, sulforaphane and antioxidant activity with phenol contents (137). Baczek-Kwinta et al. (2006) stated that SOD activity was different in different genotypes of cabbage under drought treatment. Similar results were reported by Joon-Ho and Sang (2015) and DominguezPerles et al. (2010) and indicated that there was no significant correlation between the sulforaphane content and the antioxidant activity of DPPH in Broccoli and other plants in the Brassicaceae family.

Furthermore, grouping of the varieties according to the measured parameters resulted in cluster Marathon genotype as separate group. In this study, Marathon showed relatively high values for the most of the measured traits, while the content of MDA and $\mathrm{H}_{2} \mathrm{O}_{2}$ were roughly lower than the other varieties.

\section{Conclussion}

The overall results of this study showed that the application of mannitol as a compound that decreases the osmotic potential of cells and simulates the drought stress in the in vitro condition, is a suitable compound for being used to induce the simulated drought stress in laboratory experiment. In addition, induced stress changed the content of measured traits and activities of enzymatic antioxidant. Mannitol stress reduced shoot dry weight and shoot length significantly for all genotypes but with the different rate. Marathon and Heraklion genotypes showed higher growth related traits under relatively all conditions. The response of marathon to mannitol application related to total phenolic content, flavonoid content, anthocyanin content, the activity of all enzymatic antioxidant, and the activity of DPPH was higher than the other genotypes. $\mathrm{H}_{2} \mathrm{O}_{2}$ and MDA content as the negative markers of oxidative stress were higher under mannitol stress condition. Results showed that sulforaphane content of the different genotypes under different stress levels was different. Mannitol stress increased the content of sulforaphane. Marathon showed the highest content of sulforaphane among all genotypes under both normal and stress conditions. Considering all these results together put us in touch with a suggestion that Marathon is the most proper genotypes for both cultivation and breeding aims among all studied genotypes. Heraklion genotype showed to be in second order regarding the content of measured traits after marathon with a high significant content of sulforaphane comparing other genotypes. Therefore, Heraklion might be a useful genotype being considered in line with marathon in breeding programs. Furthermore, both severe and moderate stresses increased sulforaphane amount along with phenolic compounds and antioxidant actives; therefore, it could be elucidated that moderate drought stress condition $(88 \mathrm{mM}$ concentration of Mannitol) which leaded to relatively low decreases in growth parameters comparing control condition, might be applicable for inducing higher content of sulforaphane and phenolic compounds in broccoli. More studies are needed to assess whether bioactive molecular accumulation really improve the biological / functional properties of these bioactive molecule enriched-sprouts.

Acknowledgements. The authors are grateful to the Agricultural and Natural Resources University, Sari, Mazandaran, Iran for providing the facilities for the investigations. 


\section{REFERENCES}

[1] Abou El-Magd, M., Mahmoud, A. R., Hafiz, M. M. and Ali, A. H. (2013): Effect of different levels of mineral phosphorus fertilizer and bio-phosphorus on vegetative growth, head yield and quality of broccoli. - Research Journal of Agriculture and Biological Sciences 9(5): 164-169.

[2] Aebi, H. (1984): [13] catalase in vitro. - Methods in enzymology 105: 121-126.

[3] Ainsworth, E. A., Gillespie, K. M. (2007): Estimation of total phenolic content and other oxidation substrates in plant tissues using folin-ciocalteu reagent. - Nature protocols 2(4): 875-877.

[4] Baczek-Kwinta, R., Filek, W., Grzesiak, S., Hura, T. (2006): The effect of soil drought and rehydration on growth and antioxidative activity in flag leaves of triticale. - Biologia plantarum 50: 55-60.

[5] Baxter, A., Mittler, R. Suzuki, N. (2014): Ros as key players in plant stress signalling. Journal of experimental botany 65(5): 1229-1240.

[6] Beecher, C. W. (1994): Cancer preventive properties of varieties of brassica oleracea: A review. - The American journal of clinical nutrition 59(5): 1166S-1170S.

[7] Berger, J., Palta, J., Vadez, V. (2016): Review: An integrated framework for crop adaptation to dry environments: Responses to transient and terminal drought. - Plant Science 253: 58-67.

[8] Brader, G., Mikkelsen, M. D., Halkier, B. A., Tapio Palva, E. (2006): Altering glucosinolate profiles modulates disease resistance in plants. - The Plant Journal 46(5): 758-767.

[9] Bradford, M. M. (1976): A rapid and sensitive method for the quantitation of microgram quantities of protein utilizing the principle of protein-dye binding. - Analytical biochemistry 72(1-2): 248-254.

[10] Brand-Williams, W., Cuvelier, M.-E., Berset, C. (1995): Use of a free radical method to evaluate antioxidant activity. - LWT-Food Science and Technology 28(1): 25-30.

[11] Chaves, M. M., Flexas, J., Pinheiro, C. (2009): Photosynthesis under drought and salt stress: Regulation mechanisms from whole plant to cell. - Annals of botany 103(4): 551560.

[12] Dazy, M., Jung, V., Férard, J.-F., Masfaraud, J.-F. (2008): Ecological recovery of vegetation on a coke-factory soil: Role of plant antioxidant enzymes and possible implications in site restoration. - Chemosphere 74(1): 57-63.

[13] De Dorlodot, S., Forster, B., Pagès, L., Price, A., Tuberosa, R., Draye, X. (2007): Root system architecture: Opportunities and constraints for genetic improvement of crops. Trends in plant science 12(10): 474-481.

[14] Dominguez-Perles, R., Martinez-Ballesta, M. C., Carvajal, M.,Garca-Viguera, C., Moreno, D. A. (2010): Broccoli-derived by-products-a promising source of bioactive ingredients. - Journal of Food Science 75: 383-392.

[15] Gawlik-Dziki, U. (2008): Effect of hydrothermal treatment on the antioxidant properties of broccoli (brassica oleracea var. Botrytis italica) florets. - Food chemistry 109(2): 393401.

[16] Giannopolitis, C. N., Ries, S. K. (1977): Superoxide dismutases i. Occurrence in higher plants. - Plant physiology 59(2): 309-314.

[17] Gu, Y., Guo, Q., Zhang, L., Chen, Z., Han, Y., Gu, Z. (2011): Physiological and biochemical metabolism of germinating broccoli seeds and sprouts. - Journal of Agricultural and Food Chemistry 60: 209-213.

[18] Guo, R., Yuan, G., Wang, Q. (2011): Effect of sucrose and mannitol on the accumulation of health-promoting compounds and the activity of metabolic enzymes in broccoli sprouts. - Scientia Horticulturae 128(3): 159-165.

[19] Gupta, B., Huang, B. (2014): Mechanism of salinity tolerance in plants: Physiological, biochemical, and molecular characterization. - International journal of genomics 2014. 
[20] Han, S.-K., Wagner, D. (2014): Role of chromatin in water stress responses in plants. Journal of experimental botany 65(10): 2785-2799.

[21] Heath, R. L., Packer, L. (1968): Photoperoxidation in isolated chloroplasts: I. Kinetics and stoichiometry of fatty acid peroxidation. - Archives of biochemistry and biophysics 125(1): 189-198.

[22] Hossain, M. A., Bhattacharjee, S., Armin, S.-M., Qian, P., Xin, W., Li, H.-Y., Burritt, D. J., Fujita, M., Tran, L.-S. P. (2015): Hydrogen peroxide priming modulates abiotic oxidative stress tolerance: Insights from ros detoxification and scavenging. - Frontiers in plant science 6.

[23] Hwang, J. H., Lim, S. B. (2015): Antioxidant and anticancer activities of broccoli byproducts from different cultivars and maturity stages at harvest. - Preventive Nutrition and Food Science 20(1): 8-14

[24] Lemoine, M. L., Chaves, A. R. , Martínez, G. A. (2010): Influence of combined hot air and uv-c treatment on the antioxidant system of minimally processed broccoli (brassica oleracea 1. Var. Italica). - LWT-Food Science and Technology 43(9): 1313-1319.

[25] Nakano, Y., Asada, K. (1981): Hydrogen peroxide is scavenged by ascorbate-specific peroxidase in spinach chloroplasts. - Plant and cell physiology 22(5): 867-880.

[26] Oomah, B. D., Mazza, G. (1996): Flavonoids and antioxidative activities in buckwheat. Journal of Agricultural and Food Chemistry 44(7): 1746-1750.

[27] Osakabe, Y., Yamaguchi-Shinozaki, K., Shinozaki, K., Tran, L. S. P. (2014): Aba control of plant macroelement membrane transport systems in response to water deficit and high salinity. - New Phytologist 202(1): 35-49.

[28] Pereira, F. M. V., Rosa, E., Fahey, J. W., Stephenson, K. K., Carvalho, R., Aires, A. (2002): Influence of temperature and ontogeny on the levels of glucosinolates in broccoli (brassica oleracea var. Italica) sprouts and their effect on the induction of mammalian phase 2 enzymes. - Journal of Agricultural and Food Chemistry 50(21): 6239-6244.

[29] Saed-Moucheshi, A., Pakniyat, H., Pirasteh-Anosheh, H., Azooz, M. (2014a): Role of ROS as signaling molecules in plants. - In: p, Ahmad (ed.) Reactive Oxygen Species, Antioxidant Network and Signaling in Plants. Springer Publication, New York, USA: 585-626.

[30] Saed-Moucheshi, A., Shekoofa, A., Pessarakli, M. (2014b): Reactive oxygen species (ROS) generation and detoxifying in plants. - Journal of Plant Nutrition 37(10): 15731585.

[31] Suzuki, N., Rivero, R. M., Shulaev, V., Blumwald, E., Mittler, R. (2014): Abiotic and biotic stress combinations. - New Phytologist 203(1): 32-43.

[32] Uga, Y., Sugimoto, K., Ogawa, S., Rane, J., Ishitani, M., Hara, N., Kitomi, Y., Inukai, Y., Ono, K., Kanno, N. (2013): Control of root system architecture by deeper rooting 1 increases rice yield under drought conditions. - Nature Genetics 45(9): 1097-1102.

[33] Velikova, V., Yordanov, I. and Edreva, A. (2000): Oxidative stress and some antioxidant systems in acid rain-treated bean plants: Protective role of exogenous polyamines. - Plant Science 151(1): 59-66.

[34] Vosough, A., Ghouchani, R., Saed-Moucheshi, A. (2015): Genotypic variation and heritability of antioxidant related traits in wheat landraces of iran. - Biological Forum 7(2): 43-47

[35] Yuan, Y., Chiu, L.-W., Li, L. (2009): Transcriptional regulation of anthocyanin biosynthesis in red cabbage. - Planta 230(6): 1141. 